Supplement of Hydrol. Earth Syst. Sci., 22, 4125-4143, 2018

https://doi.org/10.5194/hess-22-4125-2018-supplement

(C) Author(s) 2018. This work is distributed under

the Creative Commons Attribution 4.0 License.

(c) (1)

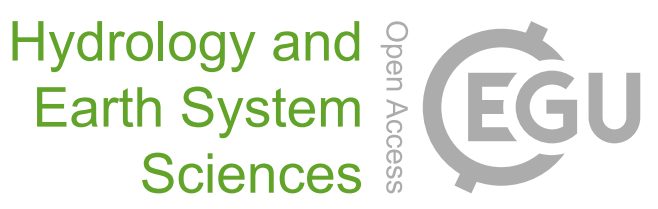

Supplement of

\title{
Multimodel assessment of climate change-induced hydrologic impacts for a Mediterranean catchment
}

Enrica Perra et al.

Correspondence to: Enrica Perra (enrica.perra@unica.it)

The copyright of individual parts of the supplement might differ from the CC BY 4.0 License. 


\begin{tabular}{|c|c|c|c|c|c|c|c|c|}
\hline \multicolumn{5}{|c|}{ Precipitation } & \multicolumn{4}{|c|}{ Temperature } \\
\hline \multirow{4}{*}{ REF } & ERC & 0.996 & 0.994 & 0.984 & ERC & 1 & 1 & 0.998 \\
\hline & 0.985 & ERE & 0.988 & 0.995 & 1 & ERE & 1 & 0.999 \\
\hline & 0.996 & 0.981 & ERM & 0.967 & 1 & 1 & ERM & 0.999 \\
\hline & 0.910 & 0.926 & 0.923 & HRC & 0.999 & 0.999 & 0.999 & HRC \\
\hline \multirow{4}{*}{ FUT } & ERC & 0.991 & 0.984 & 0.984 & ERC & 0.999 & 1 & 0.990 \\
\hline & 0.987 & ERE & 0.994 & 0.97 & 0.999 & ERE & 1 & 0.988 \\
\hline & 0.985 & 0.995 & ERM & 0.982 & 1 & 0.999 & ERM & 0.990 \\
\hline & 0.944 & 0.953 & 0.960 & HRC & 0.996 & 0.994 & 0.995 & HRC \\
\hline
\end{tabular}

Table S1: Results of the analysis of agreement for mean monthly precipitation (left) and temperature (right) among the four climate models $($ ERC $=$ ECH-RCA, ERE $=$ ECH-REM, ERM $=$ ECH-RMO, HRC $=$ HCH-RCA) for the reference $($ REF, 1971-

5 2000) and future (FUT, 2041-2070) periods. Correlation values are represented below the diagonal, while bias is reported above the diagonal. 


\begin{tabular}{|c|c|c|c|c|c|c|c|c|c|c|}
\hline \multirow{5}{*}{ ERC } & CAT & 0.811 & 0.697 & 0.848 & 0.958 & CAT & 0.330 & 0.293 & 0.475 & 0.438 \\
\hline & 0.885 & SWA & 0.877 & 0.992 & 0.885 & 0.810 & SWA & 0.985 & 0.864 & 0.907 \\
\hline & 0.897 & 0.946 & TOP & 0.833 & 0.836 & 0.881 & 0.928 & TOP & 0.785 & 0.888 \\
\hline & 0.918 & 0.939 & 0.993 & TRI & 0.898 & 0.920 & 0.945 & 0.982 & TRI & 0.844 \\
\hline & 0.864 & 0.969 & 0.877 & 0.880 & WAS & 0.823 & 0.974 & 0.914 & 0.939 & WAS \\
\hline \multirow{5}{*}{ ERE } & CAT & 0.769 & 0.660 & 0.884 & 0.981 & CAT & 0.477 & 0.428 & 0.702 & 0.767 \\
\hline & 0.854 & SWA & 0.939 & 0.907 & 0.850 & 0.673 & SWA & 0.985 & 0.827 & 0.837 \\
\hline & 0.951 & 0.937 & TOP & 0.755 & 0.756 & 0.875 & 0.932 & TOP & 0.748 & 0.782 \\
\hline & 0.958 & 0.941 & 0.987 & TRI & 0.913 & 0.861 & 0.914 & 0.987 & TRI & 0.936 \\
\hline & 0.805 & 0.953 & 0.840 & 0.860 & WAS & 0.675 & 0.973 & 0.917 & 0.909 & WAS \\
\hline \multirow{5}{*}{ ERM } & CAT & 0.646 & 0.527 & 0.765 & 0.801 & CAT & 0.206 & 0.202 & 0.335 & 0.264 \\
\hline & 0.817 & SWA & 0.858 & 0.972 & 0.891 & 0.708 & SWA & 0.999 & 0.814 & 0.940 \\
\hline & 0.949 & 0.942 & TOP & 0.820 & 0.859 & 0.842 & 0.950 & TOP & 0.795 & 0.942 \\
\hline & 0.948 & 0.925 & 0.988 & TRI & 0.948 & 0.819 & 0.962 & 0.987 & TRI & 0.833 \\
\hline & 0.699 & 0.968 & 0.870 & 0.857 & WAS & 0.698 & 0.980 & 0.946 & 0.950 & WAS \\
\hline \multirow{5}{*}{ HRC } & CAT & 0.890 & 0.751 & 0.933 & 0.945 & CAT & 0.098 & 0.105 & 0.179 & 0.146 \\
\hline & 0.889 & SWA & 0.885 & 0.993 & 0.938 & 0.810 & SWA & 0.997 & 0.732 & 0.906 \\
\hline & 0.980 & 0.916 & TOP & 0.867 & 0.901 & 0.807 & 0.994 & TOP & 0.758 & 0.934 \\
\hline & 0.982 & 0.923 & 0.993 & TRI & 0.959 & 0.840 & 0.969 & 0.979 & TRI & 0.821 \\
\hline & 0.868 & 0.970 & 0.891 & 0.906 & WAS & 0.790 & 0.978 & 0.962 & 0.910 & WAS \\
\hline
\end{tabular}

Table S2: Results of the analysis of agreement for mean monthly discharge between the five hydrologic models $($ CAT $=$ CATHY, SWA $=$ SWAT, TOP $=$ TOPKAPI, TRI $=$ tRIBS, WAS $=$ WASIM) for the reference (REF, 1971-2000) and future (FUT, 20412070) periods. Correlation values are represented below the diagonal, while bias is reported above the diagonal. 


\begin{tabular}{|c|c|c|c|c|c|c|c|c|c|c|}
\hline \multirow{5}{*}{ ERC } & CAT & 0.029 & 0.016 & 0.011 & 0.012 & CAT & 0.038 & 0.020 & 0.019 & 0.015 \\
\hline & 0.729 & SWA & 0.689 & 0.214 & 0.644 & 0.749 & SWA & 0.666 & 0.203 & 0.635 \\
\hline & 0.947 & 0.905 & TOP & 0.281 & 0.824 & 0.959 & 0.902 & TOP & 0.220 & 0.866 \\
\hline & 0.659 & 0.984 & 0.856 & TRI & 0.155 & 0.579 & 0.946 & 0.772 & TRI & 0.137 \\
\hline & 0.759 & 0.964 & 0.916 & 0.960 & WAS & 0.747 & 0.946 & 0.887 & 0.954 & WAS \\
\hline \multirow{5}{*}{ ERE } & CAT & 0.031 & 0.019 & 0.013 & 0.010 & CAT & 0.039 & 0.021 & 0.017 & 0.012 \\
\hline & 0.769 & SWA & 0.713 & 0.231 & 0.569 & 0.747 & SWA & 0.692 & 0.221 & 0.547 \\
\hline & 0.961 & 0.904 & TOP & 0.344 & 0.671 & 0.951 & 0.906 & TOP & 0.272 & 0.727 \\
\hline & 0.656 & 0.967 & 0.821 & TRI & 0.137 & 0.592 & 0.955 & 0.800 & TRI & 0.123 \\
\hline & 0.718 & 0.948 & 0.868 & 0.967 & WAS & 0.694 & 0.936 & 0.867 & 0.966 & WAS \\
\hline \multirow{5}{*}{ ERM } & CAT & 0.032 & 0.018 & 0.014 & 0.014 & CAT & 0.043 & 0.023 & 0.023 & 0.020 \\
\hline & 0.756 & SWA & 0.683 & 0.202 & 0.669 & 0.756 & SWA & 0.683 & 0.212 & 0.679 \\
\hline & 0.964 & 0.899 & TOP & 0.259 & 0.860 & 0.956 & 0.904 & TOP & 0.226 & 0.939 \\
\hline & 0.655 & 0.967 & 0.825 & TRI & 0.156 & 0.594 & 0.950 & 0.779 & TRI & 0.167 \\
\hline & 0.790 & 0.974 & 0.916 & 0.952 & WAS & 0.770 & 0.955 & 0.904 & 0.950 & WAS \\
\hline \multirow{5}{*}{ HRC } & CAT & 0.031 & 0.018 & 0.012 & 0.012 & CAT & 0.041 & 0.020 & 0.023 & 0.016 \\
\hline & 0.752 & SWA & 0.709 & 0.225 & 0.640 & 0.810 & SWA & 0.639 & 0.168 & 0.616 \\
\hline & 0.959 & 0.901 & TOP & 0.324 & 0.780 & 0.976 & 0.902 & TOP & 0.153 & 0.903 \\
\hline & 0.651 & 0.970 & 0.821 & TRI & 0.161 & 0.673 & 0.938 & 0.782 & TRI & 0.106 \\
\hline & 0.723 & 0.952 & 0.883 & 0.949 & WAS & 0.750 & 0.909 & 0.840 & 0.962 & WAS \\
\hline
\end{tabular}

Table S3: Results of the analysis of agreement for mean monthly soil water content between the five hydrologic models $(\mathrm{CAT}=$ CATHY, SWA = SWAT, TOP = TOPKAPI, TRI = tRIBS, WAS = WASIM) for the reference (REF, 1971-2000) and future (FUT, 35 2041-2070) periods. Correlation values are represented below the diagonal, while bias is reported above the diagonal. 


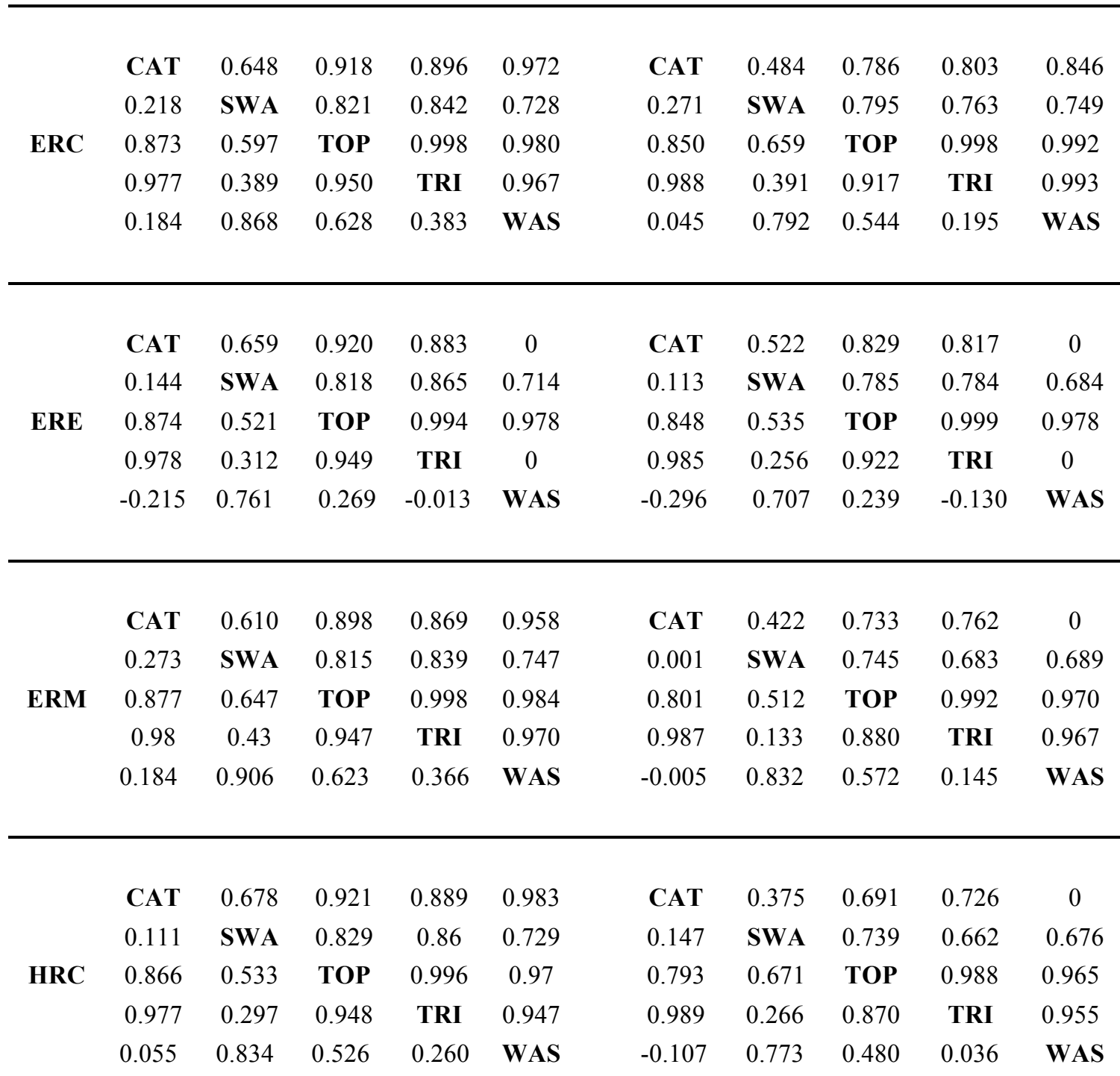

40 Table S4: Results of the analysis of agreement for mean monthly actual evapotranspiration between the five hydrologic models $($ CAT $=$ CATHY, SWA $=$ SWAT, TOP $=$ TOPKAPI, TRI $=$ tRIBS, WAS $=$ WASIM $)$ for the reference $($ REF, $1971-2000)$ and future (FUT, 2041-2070) periods. Correlation values are represented below the diagonal, while bias is reported above the diagonal. 